Article

\title{
Design, Synthesis, and Structural Characterization of Novel Diazaphenothiazines with 1,2,3-Triazole Substituents as Promising Antiproliferative Agents
}

\author{
Beata Morak-Młodawska ${ }^{1, *(\mathbb{C})}$, Krystian Pluta ${ }^{1}$, Małgorzata Latocha ${ }^{2}$, Małgorzata Jelen ${ }^{1}(\mathbb{D}$ and \\ Dariusz Kuśmierz ${ }^{2}$ \\ 1 Department of Organic Chemistry, Faculty of Pharmaceutical Sciences, The Medical University of Silesia, \\ Jagiellońska 4, 41-200 Sosnowiec, Poland; pluta@sum.edu.pl (K.P.); manowak@sum.edu.pl (M.J.) \\ 2 Department of Cell Biology, Faculty of Pharmaceutical Sciences, The Medical University of Silesia, Jedności 8, \\ 41-200 Sosnowiec, Poland; mlatocha@sum.edu.pl (M.L.); dkusmierz@sum.edu.pl (D.K.) \\ * Correspondence: bmlodawska@sum.edu.pl; Tel.: +48-32-364-16-04
}

Received: 28 October 2019; Accepted: 27 November 2019; Published: 30 November 2019

\begin{abstract}
A series of novel 1,2,3-triazole-diazphenothiazine hybrids was designed, synthesized, and evaluated for anticancer activity against four selected human tumor cell lines (SNB-19, Caco-2, A549, and MDA-MB231). The majority of the synthesized compounds exhibited significant potent activity against the investigated cell lines. Among them, compounds $\mathbf{1 d}$ and $4 \mathbf{c}$ showed excellent broad spectrum anticancer activity, with $\mathrm{IC}_{50}$ values ranging from 0.25 to $4.66 \mu \mathrm{M}$ and 0.25 to $6.25 \mu \mathrm{M}$, respectively. The most promising compound $\mathbf{1 d}$, possessing low cytotoxicity against normal human fibroblasts NHFF, was used for gene expression analysis using reverse transcription-quantitative real-time PCR (RT-qPCR). The expression of H3, TP53, CDKN1A, BCL-2, and BAX genes revealed that these compounds inhibited the proliferation in all cells $(H 3)$ and activated mitochondrial events of apoptosis $(B A X / B C L-2)$.
\end{abstract}

Keywords: diazaphenothiazines; 1,2,3-triazole ring; antiproliferative activity; intracellular apoptosis pathway; $H 3$; TP53; $C D K N 1 A ; B A X / B C L-2$ ratio

\section{Introduction}

The phenothiazines were the first synthetic agents to be used successfully in psychiatry as neuroleptics. The properties of this group of heterocyclic compounds was discovered in the 1950s and provoked revolutions in contemporary medicine [1]. Currently, they are also well-known clinically as substances with antibacterial, antiviral, anti-inflammatory, and anti-tuberculosis properties. There have also been numerous reports on the antitumor activity of these drugs [2-6]. Neuroleptic phenothiazines became the foundation for numerous studies on modified phenothiazine systems that show a wide spectrum of biological activities, including: anticancer, antibacterial, antifungal, antiviral, and antiparasitic. This rich scientific material is the subject of numerous publications that can be found in the world literature every year [7-16].

The replacement of two benzene rings with the pyridine rings leads to dipyridothiazines. These diazaphenothiazines were found to be an excellent scaffold for novel anticancer agents with high safety profile. We synthesized dipyridothiazines of the 1,6-, 1,8-, 2,7-, and 3,6-diazaphenothiazine structures with varied alkyl, aryl, heteroaryl, dialkylaminoalkyl, amidoalkyl, sulfonamidoalkyl, and dialkylaminoalkynyl substituents at the thiazine nitrogen atom. Some selected compounds showed very promising anticancer, immunosuppressant, and antioxidant activities, and low toxicity [17-25].

The 1,2,3-triazoles, due to their unique chemical and structural properties, have received much attention over the past decades, and have been well-recognized for their broad range of pharmacological 
properties. This heterocycle has been well exploited for the generation of many medicinal scaffords exhibiting anti-HIV, anticancer, antimicrobial, and antidepressant activities [26-28].

Several triazoles (e.g., cefatrizine, tazobactum) have been used in medicine as $\beta$-lactam antibiotics [29]. Additionally 1,2,3-triazoles can form hydrogen bonds, which play important roles for bioavailability and solubility [30]. The triazole ring is the biological linker and exhibits bioisosteric effects on various heteroaromatic and aromatic rings [31-33].

The association of the potency of dipyridothiazine and 1,2,3-triazole pharmacophores under the same molecular frame was the target of our on-going idea for rational design of new biologically active compounds. The bioactive dipyridothiazines (1,6-, 1,8-, 2,7-, and 3,6-diazaphenothiazine 1-4) were combined with the 1,2,3-triazole system, using 1,3-dipolar cycloaddition reaction between 2-propynyl derivatives of dipyridothiazines (1-4)a and some selected organic azides.

For the triazole hybrids, the anticancer action on the selected cancer cell lines: glioblastoma SNB-19, colorectal carcinoma Caco-2, lung cancer A549, and breast cancer MDA-MB231 were investigated. The cytotoxicity was determined towards normal human fibroblasts NHDF. In order to understand the mechanism of action and the effects on cancer biology, the expression of H3, TP53, CDKN1A, BCL-2 and $B A X$ genes was detected for the most active compound by the RT-qPCR method.

\section{Results}

\subsection{Chemistry}

The synthetic strategy applied for the preparation of the new triazole hybrids of diazphenothiazines was founded on selection of dipyridothiazines 1-4 as the building block laid open for assembling the 1,2,3-triazole ring, as presented in Scheme 1.<smiles>CC1=C(C)Nc2ccccc2Sc2ccccc2N1</smiles>

$\mathrm{H}$<smiles>c1cc2c(cn1)Nc1ccncc1S2</smiles>

3 2,7-diazaphenothiazine<smiles>c1cnc2c(c1)Nc1ccncc1S2</smiles>

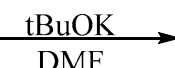
DMF<smiles>C#CCN1c2ccccc2Sc2ccccc21</smiles>
$1 \mathrm{a}-4 \mathbf{a}$<smiles>CC(C)(C)O</smiles>

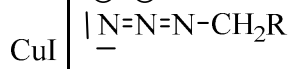
toluene, $60^{\circ} \mathrm{C}$<smiles>[R]Cc1ccc([Y])cc1</smiles><smiles>[X]c1ccc(Cn2cc(CN3c4ccccc4Sc4ccccc43)nn2)cc1</smiles>

$$
\begin{aligned}
& X=H 1 b-4 b \\
& X=F \quad 1 c-4 c \\
& X=C l 1 d-4 d \\
& X=C N 1 e-4 e
\end{aligned}
$$$$
\text { 1f - } 4 f
$$

Scheme 1. Synthesis of 1,2,3-triazole derivatives of dipyridothiazines (1-4)b-f. 
The starting materials: 10H-1,6-, 1,8-, 2,7-, and 3,6-diazaphenothiazines 1-4, were transformed with 2-propynyl bromide into the propynyl derivatives (1-4)a according to the described synthesis [20-23], and further using "click chemistry"-1,3-dipolar cycloadditon (with selected azides, in toluene, in the presence of $\mathrm{CuI}$ as a catalyst) into substituted triazole derivatives of diazaphenothiazines (1-4)b-f in good yields (67-82\%).

Bearing in mind the fact of significant biological activities of triazole systems with various benzylic and phenyl substituents [34-36], the following selected azides (benzyl azide, p-fluorobenzyl azide, $p$-chlorobenzyl azide, $p$-cyanobenzyl azide, and phenylthiometyl azide) were selected for the 1,3-dipolar cycloaddition.

The identification of the product structure was based on ${ }^{1} \mathrm{H},{ }^{13} \mathrm{C}$ NMR spectra, 2D NMR experiments: COSY and ROESY, and mass spectrometry HRMS.

The crude products of the reactions were separated by column chromatography to obtain pure final derivatives $(\mathbf{1}-\mathbf{4}) \mathbf{b}-\mathbf{f}$ with good yields.

On the basis of previous studies, the reaction of 2-propynyl derivatives with organic azides can led to 1,4- and/or 1,5-regioisomers [26,28]. Bearing in mind the literature, using a CuI catalyst, a 1,4 regioisomer is obtained selectively $[28,34]$. Nevertheless, the structure of the products received had to be unambiguously confirmed. For this reason, the 2D NMR ROESY experiment of compound $\mathbf{1 b}$ was carried out (Figure 1, Table 1, Supplementary Material). For triazole hybrid 1b, protons of the benzylic group $\left(\mathrm{CH}_{2} \mathrm{~N}_{\mathrm{Tr}}\right.$ where $\mathrm{Tr}$-triazole ring) intercorrelated with aromatic proton of the 1,2,3-triazole ring and orto-protons of the benzene ring. However, if a substitution product of 1,5-regioisomer 1B was formed in the reaction, intercorrelation between the $\mathrm{CH}_{2}$ group at the thiazine nitrogen atom $\left(\mathrm{CH}_{2} \mathrm{~N}_{\mathrm{Th}}\right.$ where Th-thiazine ring) with the $\mathrm{CH}_{2}$ group at the triazole ring $\left(\mathrm{CH}_{2} \mathrm{~N}_{\mathrm{Tr}}\right)$ would be expected but was not observed.

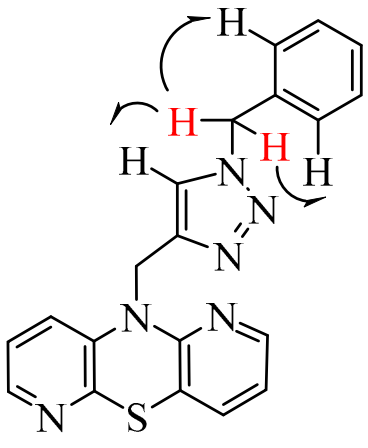

$\mathbf{1 b}$

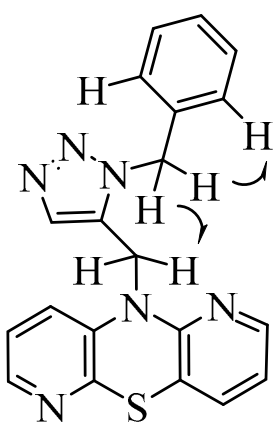

$1 \mathrm{~B}$

Figure 1. The ROESY experiment for triazole derivative $\mathbf{1 b}$.

Table 1. The ${ }^{1} \mathrm{H}$ NMR chemical shifts and proton-proton correlations (ROESY and COSY experiments) for compound $\mathbf{1 b}$.

\begin{tabular}{ccc}
\hline${ }^{1} \mathbf{H}$ NMR & ROESY & COSY \\
\hline $5.22 \mathrm{CH}_{2} \mathrm{~N}_{\mathrm{Th}}$ & $5.22 / 7.46$ & $5.22 / 7.61$ \\
$5.49 \mathrm{CH}_{2} \mathrm{~N}_{\mathrm{Tr}}$ & $5.49 / 7.23 / 7.61$ & $5.49 / 7.23 / 7.61$ \\
$6.76 \mathrm{H}_{3}$ & $6.76 / 7.23 / 7.92$ & $6.76 / 7.23 / 7.92$ \\
$6.93 \mathrm{H}_{8}$ & $6.93 / 7.46$ & $6.93 / 7.46 / 7.92$ \\
$7.23 o-\mathrm{H}_{\mathrm{Ph}}, 1 \mathrm{H}_{4}$ & $7.23 / 6.76 / 7.35$ & $7.23 / 6.76 / 7.35 / 7.92$ \\
$7.35 \mathrm{~m}, p-\mathrm{H}_{\mathrm{Ph}}$ & $7.35 / 7.23$ & $7.35 / 7.23$ \\
$7.46 \mathrm{H}_{9}$ & $7.61 / 5.22$ & $7.46 / 6.93 / 7.92$ \\
$7.61 \mathrm{H}_{\mathrm{Tr}}$ & $7.61 / 5.49 / 7.23$ & $7.61 / 5.22$ \\
$7.92 \mathrm{H}_{7}+\mathrm{H}_{2}$ & $7.92 / 6.76 / 6.93$ & $7.92 / 6.76 / 6.93 / 7.23 / 7.46$ \\
\hline
\end{tabular}


This we consider to be proof of the presence of 1,4-disubstituted 1,2,3-triazole ring. The full correlation between the neighbouring protones showed COSY spectra (Table 1, Supplementary Material).

\subsection{Anticancer Activity}

Encouraged by our previous promising results in the field of antiproliferative activity of dipyridothiazines [17-25] as well as by the good anticancer activity of the 1,2,3-triazoles [26-33], we decided to combine the biological potentials of dipyridothiazines and triazole moieties, hoping to obtain compounds with better activity. The antiproliferative activity of all 20 new 10-substituted 1,2,3-triazole derivatives of dipyridothiazines was tested in vitro against cancer cell lines: glioblastoma SNB-19, colorectal carcinoma Caco-2, lung cancer A549, and breast cancer MDA-MB231 using the WST-1 assay. Normal human fibroblasts NHDF were used as a control. Cisplatin was used as a positive control to induce cell death. The results of the cytotoxicity studies were summarized in Table 2.

Table 2. Anticancer activities $\mathrm{IC}_{50}(\mu \mathrm{M})$ of 10-substituted 3,6-diazaphenothiazines (1b-4f) against glioblastoma SNB-19, colorectal carcinoma Caco-2, lung cancer A549, breast cancer MDA-MB231 cells and normal human fibroblasts NHDF.

\begin{tabular}{|c|c|c|c|c|c|}
\hline \multirow{2}{*}{ No } & \multicolumn{5}{|c|}{ Anticancer Activity $\mathrm{IC}_{50}(\mu \mathrm{M})$} \\
\hline & SNB-19 & Caco-2 & A549 & MDA-MB231 & NHDF \\
\hline $1 b$ & $69.15+/-3.14$ & $19.92+/-2.71$ & $>100$ & $13.66+/-1.16$ & $>100$ \\
\hline 1c & $5.72+/-1.44$ & $8.10+/-0.92$ & $29.12+/-2.59$ & $7.82+/-1.32$ & $>100$ \\
\hline 1d & $4.66+/-1.16$ & $0.25+/-0.02$ & $0.25+/-0.01$ & $0.51+/-0.09$ & $>100$ \\
\hline 1e & $9.65+/-1.99$ & $79.65+/-4.12$ & $1.79+/-0.62$ & $25.79+/-1.32$ & $>100$ \\
\hline 1f & $>100$ & $>100$ & $>100$ & $68.68+/-5.12$ & $>100$ \\
\hline $2 b$ & $41.91+/-3.15$ & $>100$ & $77.44+/-3.22$ & $56.34+/-5.72$ & $>100$ \\
\hline $2 c$ & $55.23+/-5.78$ & $43.61+/-6.52$ & $60.23+/-2.77$ & $9.21+/-3.01$ & $>100$ \\
\hline $2 d$ & $59.87+/-9.99$ & $11.42+/-2.13$ & $1.82+/-0.71$ & $4.71+/-0.22$ & $42.95+/-5.11$ \\
\hline $2 e$ & $10.62+/-1.11$ & $34.38+/-2.72$ & $63.75+/-2.78$ & $12.04+/-2.21$ & $>100$ \\
\hline $2 f$ & $56.34+/-5.72$ & $32.55+/-8.21$ & $46.80+/-2.73$ & $12.04+/-6.11$ & $>100$ \\
\hline $3 b$ & $2.04+/-0.21$ & $0.26+/-0.01$ & $0.26+/-0.11$ & $0.77+/-0.10$ & $22.66+/-1.32$ \\
\hline $3 c$ & $57.92+/-4.96$ & $29.87+/-5.21$ & $27.23+/-2.16$ & $25.66+/-4.11$ & $70.61+/-12.21$ \\
\hline $3 d$ & $31.31+/-10.99$ & $33.07+/-9.18$ & $17.91+/-1.11$ & $4.26+/-0.15$ & $>100$ \\
\hline $3 e$ & $26.80+/-1.72$ & $10.02+/-0.99$ & $30.07+/-3.21$ & $3.50+/-0.35$ & $49.47+/-1.11$ \\
\hline $3 f$ & $32.58+/-2.77$ & $14.54+/-1.22$ & $0.65+/-0.10$ & $0.64+/-0.10$ & $52.90+/-3.21$ \\
\hline $4 b$ & $20.80+/-1.96$ & $57.90+/-2.15$ & $60.62+/-3.52$ & $7.87+/-1.11$ & $>100$ \\
\hline $4 c$ & $6.25+/-0.62$ & $0.25+/-0.02$ & $0.25+/-0.01$ & $1.97+/-0.13$ & $2.85+/-0.34$ \\
\hline $4 d$ & $14.87+/-1.70$ & $42.06+/-2.99$ & $53.99+/-10.72$ & $13.44+/-1.22$ & $>100$ \\
\hline $4 e$ & $53.62+/-3.72$ & $74.28+/-4.72$ & $42.69+/-13.72$ & $38.77+/-2.12$ & $>100$ \\
\hline $4 f$ & $44.83+/-13.62$ & $67.52+/-11.87$ & $>100$ & $44.33+/-11.79$ & $>100$ \\
\hline Cisplatin & $3.73+/-0.62$ & $10.53+/-1.52$ & $0.60+/-0.11$ & $3.13+/-0.24$ & $63.87+/-1.32$ \\
\hline
\end{tabular}

The most active derivative in relation to all tumor groups was compound $\mathbf{1 d}$, which structure contained the $p$-chlorobenzyl substituent $\left(\mathrm{IC}_{50}=0.25-4.66 \mu \mathrm{M}\right)$. This compound also showed relatively low cytotoxicity in relation to normal fibroblasts NHDF due to the vacancy of the examined controls. This compound showed higher activity compared to cisplatin $\left(\mathrm{IC}_{50}=0.60-10.53 \mu \mathrm{M}\right)$. In the 1,6-diazaphenothiazine group, derivative $1 \mathrm{c}$ with the $p$-fluorobenzyl substituent was also characterized by high anticancer activity in relation to SNB-19 and MDA-MB231 cell lines $\left(\mathrm{IC}_{50}=5.72-7.82 \mu \mathrm{M}\right)$. 1,8-Diazaphenothiazine $\mathbf{2} \mathbf{d}$ (with the $p$-chlorobenzyl substituent in the 1,2,3-tiazole system) showed high cytotoxic activity in relation to lung cancer cell lines A-549 $\left(\mathrm{IC}_{50}=1.82 \mu \mathrm{M}\right)$. This compound was also active against Caco-2 $\left(\mathrm{IC}_{50}=11.42 \mu \mathrm{M}\right)$ and MDA-MB231 $\left(\mathrm{IC}_{50}=4.71 \mu \mathrm{M}\right)$ cell lines. In the group of 1,8-diazaphenothiazines derivative $2 \mathbf{e}$ with the $p$-cyanobenzyl substituent stood out. The compound showed significant activity against glioblastoma SNB-19 $\left(\mathrm{IC}_{50}=10.62 \mu \mathrm{M}\right)$ and breast cancer MDA-MB231 $\left(\mathrm{IC}_{50}=12.04 \mu \mathrm{M}\right.$ ). 2,7-Diazaphenothiazine $\mathbf{3 b}$ (with the benzyl substituent in 
1,2,3-tiazole system) showed high activity against all investigated cancer cell lines $\left(\mathrm{IC}_{50}=0.26-2.07 \mu \mathrm{M}\right)$. On the other hand derivative $3 \mathbf{f}$ (with the phenyltiomethyl substituent in 1,2,3-triazole system) acted selectively towards A549 and MDA-MB231 cell lines. In the 3,6-diazaphenothiazine group, the highest anticancer activity was characterized by $4 \mathrm{c}$ derivative with the $p$-fluorobenzyl substituent $\left(\mathrm{IC}_{50}=0.25-6.25 \mu \mathrm{M}\right)$. This compound showed high cytotoxicity in relation to all cancer cells lines, however, it also showed high cytotoxicity in relation to normal human fibroblasts $\left(\mathrm{IC}_{50}=2.85 \mu \mathrm{M}\right)$.

Most of the tested compounds (1-4)b-f were characterized by significant antiproliferative activity in relation to the examined cancer cell lines and relatively weak cytotoxicity with respect to normal human fibroblasts. This activity was largely dependent on the location of nitrogen atoms in isomeric dipyridothiazines as well as on the substituent in the benzene ring of the 1,2,3-tiazole system.

\subsection{Apoptosis Assay}

10-((1-(4-Chlorobenzyl)-1H-1,2,3-triazol-4-yl)methyl)-1,6-diazaphenothiazine (1d)—the most active compound-was selected to study the mechanism of anticancer activity using RT-qPCR. In this method the gene transcriptional activities of proliferation marker (H3), cell cycle regulator (TP53 and CDKNIA), and intracellular apoptosis pathway (BCL-2 and BAX) were analyzed. The results obtained on four cancer cell lines are collected in Table 3. It has been known that the growth, metabolism, and eventual death of the cells in the body are controlled by hundreds of genes working together [35-37]. The gene encoding histone $H 3$ plays a crucial role in regulation of the expression of the genetic information included in the DNA. Compound 1d strongly changed the mRNA copy number of the histone $H 3$ gene in all cancer lines which has an influence on the modification of the chromatin structure in the cells.

Table 3. The influence of compound 1d on expression of genes encoding H3, TP53, CDKN1A, BCL-2, and $B A X$ in glioblastoma SNB-19, colorectal carcinoma Caco-2, lung cancer A549, and breast cancer MDA-MB231 cells.

\begin{tabular}{|c|c|c|c|c|c|}
\hline \multirow{2}{*}{ gene } & & SNB-19 & Caco-2 & A549 & MDA-MB231 \\
\hline & & \multicolumn{4}{|c|}{ 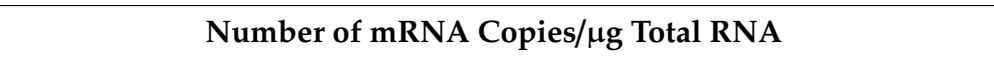 } \\
\hline \multirow{2}{*}{$H 3$} & control & $143+/-41$ & $11+/-3$ & $23+/-13$ & $2+/-1$ \\
\hline & $1 d$ & $52+/-11$ & $45+/-3$ & $18+/-5$ & $8+/-6$ \\
\hline \multirow{2}{*}{ TP53 } & control & $198+/-52$ & $65+/-24$ & $49+/-17$ & $56+/-16$ \\
\hline & $1 d$ & $128+/-24$ & $193+/-16$ & $54+/-19$ & $46+/-20$ \\
\hline \multirow{2}{*}{ CDKN1A } & control & $698+/-34$ & $266+/-49$ & $1012+/-143$ & $710+/-486$ \\
\hline & $1 d$ & $308+/-51$ & $260+/-21$ & $969+/-137$ & $577+/-308$ \\
\hline \multirow{2}{*}{$B C L-2$} & control & $70604+/-7852$ & $673718+/-52998$ & $22485+/-4794$ & $207802+/-15572$ \\
\hline & $1 d$ & $17497+/-4607$ & $723708+/-217134$ & $15631+/-2296$ & $16495+/-4607$ \\
\hline \multirow{2}{*}{$B A X$} & control & $995+/-98$ & $1062+/-141$ & $1181+/-132$ & $835+/-63$ \\
\hline & $1 d$ & $220+/-18$ & $969+/-264$ & $835+/-231$ & $221+/-16$ \\
\hline \multirow{2}{*}{$B C L-2 / B A X$} & control & 71 & 634 & 19 & 249 \\
\hline & $1 d$ & 80 & 743 & 18 & 80 \\
\hline
\end{tabular}

It is well known, that cellular stresses such as the DNA damage and oncogene activation can change the expression of the TP53 gene encoding the p53 protein which is called the guardian of the genome of the cell. The p53 protein influences cell cycle arrest by changing the expression of CDKN1A gene encoding the p21 protein. The essential function of the p21 protein is to arrest cell cycle progression by inhibiting the activity of cyclin-dependent kinases [37-39].

Compound 1d significantly interfered with the amount of mRNA copies of TP53 in all investigated cancer lines. There was also a strong decline of $C D K N 1 A$ copies in all cancer cells what suggests the possibility of participation in cell cycle arrest. 
In biochemical processes of cells, the p53 protein determines the standard balance between the expression of the proapoptotic $B A X$ gene and the antiapoptotic $B C L-2$ gene. The balance between these proteins determines whether the cell is undergoing apoptosis or interrupting the process. It is believed that the main mechanism of action of the $B C L-2$ family of proteins regulates the release of cytochrome c from mitochondria by changing the mitochondrial membrane permeability $[40,41]$.

Compound 1d remarkably reduced the expression of BCL-2 in SNB-19, A549 and MDA-MB231 cancer lines, but there was a slight increase in Caco-2 cell line. On the other hand, a decrease of the expression of $B A X$ was observed in all investigated cancer cell lines.

Analysis of the gene expression ratio $B C L-2 / B A X$ in MDA-MB231 cell showed activation of the mitochondrial apoptosis as the internal pathway of cell death. Transcriptional activity of these genes in the SNB-19, Caco-2, and A549 cells suggests a different way of cell death, possibly associated with the external pathway of apoptosis. The presented results indicate the possibility of a two-way mechanism of apoptosis caused by the hybrid of diazaphenothiazine with the 1,2,3-triazole system. However, further research is required to fully confirm this hypothesis.

\section{Materials and Methods}

\subsection{Chemistry}

The standard NMR spectra were recorded on Bruker Avance spectrometers $\left({ }^{1} \mathrm{H}\right.$ at $600 \mathrm{MHz},{ }^{13} \mathrm{C}$ at $150 \mathrm{MHz}$ ) in $\mathrm{CDCl}_{3}$. Two-dimensional COSY and ROESY, spectra of selected compounds were recorded on a Bruker Avance spectrometer at $600 \mathrm{MHz}$. The HRMS spectra (EI-electro impact ionisation) were run on a Brucker Impact II. The thin layer chromatography was performed on aluminium oxide $60 \mathrm{~F}_{254}$ neutral (type E) (Merck 1.05581) with $\mathrm{CHCl}_{3}-\mathrm{EtOH}(10: 1 \mathrm{v} / \mathrm{v})$ as eluent.

10H-1,6-diazaphenothiazine (1), 10H-1,8-diazaphenothiazine (2), 10H-2,7-diazaphenothiazine (3) and 10H-3,6-diazaphenothiazine (4) were obtained and were transformed into 10-propynyl derivatives (1-4)a according to previously described methods [18,20-23].

General Procedure for Synthesis of Compounds (1-4)b. To a solution of 10-propynyl diazaphenothiazine (1-4)a $(0.120 \mathrm{mg}, 0.5 \mathrm{mmol})$ and copper iodide (I) (catalytic amount) in dry toluene $(5 \mathrm{~mL})$, a corresponding organic azide $(0.510 \mathrm{mmol})$ was added. The reaction mixture was stirred and heated at $70{ }^{\circ} \mathrm{C}$ for $48 \mathrm{~h}$. Then the solvent mixture was distilled under reduced pressure. The dry residue was dissolved in $\mathrm{CHCl}_{3}$ and purified by column chromatography (aluminum oxide 90 active neutral, Merck 1.01077.2000, $\mathrm{CHCl}_{3}$ as eluent) to give pure triazole derivatives $\mathbf{1 b}-\mathbf{4 f}$ :

10-[(1-Benzyl-1H-1,2,3-triazol-4-yl)-methyl]-1,6-diazaphenothiazine (1b). (136 mg, 76\%) brown oil. ${ }^{1} \mathrm{H} \mathrm{NMR}_{\left(\mathrm{CDCl}_{3}\right)}$ ): $5.22\left(\mathrm{~s}, 2 \mathrm{H}, \mathrm{CH}_{2} \mathrm{~N}_{\mathrm{Th}}\right), 5.49\left(\mathrm{~s}, 2 \mathrm{H}, \mathrm{CH}_{2} \mathrm{~N}_{\mathrm{Tr}}\right), 6.76\left(\mathrm{dd}, J=7.2 \mathrm{~Hz}, J=4.8 \mathrm{~Hz} 1 \mathrm{H}, \mathrm{H}_{3}\right)$, $6.93\left(\mathrm{dd}, J=7.2 \mathrm{~Hz}, J=4.8 \mathrm{~Hz}, 1 \mathrm{H}, \mathrm{H}_{8}\right), 7.23\left(\mathrm{~m}, 3 \mathrm{H}, 2 \mathrm{H}_{\mathrm{Ph}}, 1 \mathrm{H}_{4}\right), 7.35\left(\mathrm{~m}, 3 \mathrm{H}, 3 \mathrm{H}_{\mathrm{Ph}}\right), 7.46(\mathrm{~d}, J=7.2 \mathrm{~Hz}$, $\left.1 \mathrm{H}, \mathrm{H}_{9}\right), 7.61\left(\mathrm{~s}, 1 \mathrm{H}, \mathrm{H}_{\mathrm{Tr}}\right), 7.92\left(\mathrm{~m}, 2 \mathrm{H}, \mathrm{H}_{7}+\mathrm{H}_{2}\right) .{ }^{13} \mathrm{C}$ NMR: 41.94, 54.13, 116.53, 118.39, 121.57, 122.21, $124.06,127.87,128.68,129.07,134.46,134.65,138.52,142.69,143.89,144.82,144.88,152.12$. HRMS (EI) $m / z$ for: $\left[\mathrm{C}_{20} \mathrm{H}_{16} \mathrm{~N}_{6} \mathrm{~S}+\mathrm{H}\right]$ calc. 373.1235 Found: 373.1225

10-[(1-(4-Fluorobenzyl)-1H-1,2,3-triazol-4-yl)methyl]-1,6-diazaphenothiazine (1c). (140 mg, 74\%) yellow oil. ${ }^{1} \mathrm{H} \mathrm{NMR}\left(\mathrm{CDCl}_{3}\right)$ 8: $5.22\left(\mathrm{~s}, 2 \mathrm{H}, \mathrm{CH}_{2} \mathrm{~N}_{\mathrm{Th}}\right), 5.46\left(\mathrm{~s}, 2 \mathrm{H}, \mathrm{CH}_{2} \mathrm{~N}_{\mathrm{Tr}}\right), 6.77\left(\mathrm{~m}, 1 \mathrm{H}, \mathrm{H}_{3}\right), 6.93\left(\mathrm{~m}, 1 \mathrm{H}, \mathrm{H}_{8}\right)$, $7.05\left(\mathrm{~m}, 2 \mathrm{H}, 2 \mathrm{H}_{\mathrm{Ph}}\right), 7.23\left(\mathrm{~m}, 3 \mathrm{H}, 2 \mathrm{H}_{\mathrm{Ph}}, 1 \mathrm{H}_{4}\right), 7.45\left(\mathrm{~m}, 1 \mathrm{H}, 1 \mathrm{H}_{9}\right), 7.59\left(\mathrm{~s}, 1 \mathrm{H}, \mathrm{H}_{\mathrm{Tr}}\right), 7.94\left(\mathrm{~m}, 2 \mathrm{H}, \mathrm{H}_{7}+\mathrm{H}_{2}\right)$. ${ }^{13}$ C NMR: 41.94, 53.40, 115.73, 116.01, 116.53, 118.40, 121.49, 122.17, 123.91, 127.78, 129.83, 130.00, $130.05,134.46,138.47,142.79,143.93,144.79,145.03,152.12$. HRMS (EI) $m / z$ for: $\left[C_{20} \mathrm{H}_{15} \mathrm{FN}_{6} \mathrm{~S}+\mathrm{H}\right]$ calc. 391.1141 Found: 391.1145.

10-[(1-(4-Chlorobenzyl)-1H-1,2,3-triazol-4-yl)methyl]-1,6-diazaphenothiazine (1d). (139 mg, 70\%). brown oil. ${ }^{1} \mathrm{H} \mathrm{NMR}\left(\mathrm{CDCl}_{3}\right) \delta: 5.21\left(\mathrm{~s}, 2 \mathrm{H}, \mathrm{CH}_{2} \mathrm{~N}_{\mathrm{Th}}\right), 5,46\left(\mathrm{~s}, 2 \mathrm{H}, \mathrm{CH}_{2} \mathrm{~N}_{\mathrm{Tr}}\right), 6.77\left(\mathrm{~m}, 1 \mathrm{H}, \mathrm{H}_{3}\right), 6.92(\mathrm{~m}, 1 \mathrm{H}$, $\left.\mathrm{H}_{8}\right), 7.18\left(\mathrm{~m}, 2 \mathrm{H}, 2 \mathrm{H}_{\mathrm{Ph}}\right), 7.23\left(\mathrm{~m}, 1 \mathrm{H}, \mathrm{H}_{4}\right), 7.32\left(\mathrm{~m}, 2 \mathrm{H}, 2 \mathrm{H}_{\mathrm{Ph}}\right), 7.44\left(\mathrm{~m}, 1 \mathrm{H}, \mathrm{H}_{9}\right), 7.61\left(\mathrm{~s}, 1 \mathrm{H}, 1 \mathrm{H}_{\mathrm{Tr}}\right), 7.94$ $\left(\mathrm{m}, 2 \mathrm{H}, \mathrm{H}_{7}+\mathrm{H}_{2}\right) .{ }^{13} \mathrm{C}$ NMR: 41.91, 54.40, 116.61, 118.43, 121.46, 122.19, 124.02, 129.25, 129.28, 133.13, $134.47,134.72,138.46,142.80,143.92,144.82,145.10,152.09$. HRMS (EI) $m / z$ for: $\left[\mathrm{C}_{20} \mathrm{H}_{15} \mathrm{ClN}_{6} \mathrm{~S}+\mathrm{H}\right]$ calc. 407.0846 Found: 407.0836 
10-[(1-(4-Cyanobenzyl)-1H-1,2,3-triazol-4-yl)methyl]-1,6-diazaphenothiazine (1e). (130 mg, 67\%) brown

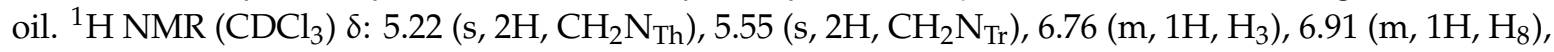
$7.21\left(\mathrm{~m}, 1 \mathrm{H}, \mathrm{H}_{4}\right), 7.28\left(\mathrm{~m}, 2 \mathrm{H}, 2 \mathrm{H}_{\mathrm{Ph}}\right), 7.41\left(\mathrm{~m}, 1 \mathrm{H}, \mathrm{H}_{9}\right), 7.63\left(\mathrm{~m}, 2 \mathrm{H}, 2 \mathrm{H}_{\mathrm{Ph}}\right), 7.68\left(\mathrm{~s}, 1 \mathrm{H}, \mathrm{H}_{\mathrm{Tr}}\right), 7.93(\mathrm{~m}, 2 \mathrm{H}$, $\left.\mathrm{H}_{7}+\mathrm{H}_{2}\right) .{ }^{13} \mathrm{C}$ NMR: 41.85, 53.36, 112.63, 116.60, 118.41, 120.29, 121.37, 122.18, 124.43, 128.29, 129.07, $132.84,134.50,138.41,142.82,143.92,144.81,145.32,152.00$. HRMS (EI) $m / z$ for: $\left[\mathrm{C}_{21} \mathrm{H}_{15} \mathrm{~N}_{7} \mathrm{~S}+\mathrm{H}\right]$ calc. 398.1189 Found: 398.1176

10-[(1-Phenylthiomethyl-1H-1,2,3-triazolo-4-ylo)methyl]-1,6-diazaphenothiazine (1f). (150 mg, 75\%) brown oil. ${ }^{1} \mathrm{H} \mathrm{NMR}\left(\mathrm{CDCl}_{3}\right) \delta: 5.18\left(\mathrm{~s}, 2 \mathrm{H}, \mathrm{CH}_{2} \mathrm{~N}_{\mathrm{Th}}\right), 5.55\left(\mathrm{~s}, 2 \mathrm{H}, \mathrm{CH}_{2} \mathrm{~N}_{\mathrm{Tr}}\right), 6.79\left(\mathrm{~m}, 1 \mathrm{H}, \mathrm{H}_{3}\right), 6.92(\mathrm{~m}$, $\left.1 \mathrm{H}, \mathrm{H}_{8}\right), 7.22\left(\mathrm{~m}, 6 \mathrm{H}, 5 \mathrm{H}_{\mathrm{Ph}}, \mathrm{H}_{9}\right), 7.34\left(\mathrm{~m}, 1 \mathrm{H}, \mathrm{H}_{4}\right), 7.49\left(\mathrm{~s}, 1 \mathrm{H}, 1 \mathrm{H}_{\mathrm{Tr}}\right), 7.92\left(\mathrm{~m}, 2 \mathrm{H}, \mathrm{H}_{7}+\mathrm{H}_{2}\right) .{ }^{13} \mathrm{C} \mathrm{NMR}$ : 41.77, 54.05, 116.49, 118.43, 121.38, 122.17, 123.70, 128.88, 129.36, 131.51, 132.89, 134.48, 138.43, 142.76, 143.91, 144.87, 144.91, 152.01. HRMS (EI) $m / z$ for: $\left[\mathrm{C}_{20} \mathrm{H}_{16} \mathrm{~N}_{6} \mathrm{~S}_{2}+\mathrm{H}\right]$ calc. 405.0956 Found: 405.0957

10-[(1-Benzyl-1H-1,2,3-triazol-4-yl)-methyl]-1,8-diazaphenothiazine (2b). (135 mg, 75\%) brown oil. ${ }^{1} \mathrm{H}$ $\operatorname{NMR}\left(\mathrm{CDCl}_{3}\right) \delta: 5.31\left(\mathrm{~s}, 2 \mathrm{H}, \mathrm{CH}_{2} \mathrm{~N}_{\mathrm{Th}}\right), 5.50\left(\mathrm{~s}, 2 \mathrm{H}, \mathrm{CH}_{2} \mathrm{~N}_{\mathrm{Tr}}\right), 6.78\left(\mathrm{~m}, 1 \mathrm{H}, \mathrm{H}_{3}\right), 6.93\left(\mathrm{~m}, 1 \mathrm{H}, \mathrm{H}_{6}\right), 7.19$ $\left(\mathrm{m}, 1 \mathrm{H}, \mathrm{H}_{4}\right), 7.20\left(\mathrm{~m}, 2 \mathrm{H}, \mathrm{H}_{\mathrm{Ph}}\right), 7.35\left(\mathrm{~m}, 3 \mathrm{H}, \mathrm{H}_{\mathrm{Ph}}\right), 7.51\left(\mathrm{~s}, 1 \mathrm{H}, \mathrm{H}_{\mathrm{Tr}}\right), 7.96\left(\mathrm{~m}, 1 \mathrm{H}, \mathrm{H}_{2}\right), 8.04\left(\mathrm{~m}, 1 \mathrm{H}, \mathrm{H}_{7}\right)$, 8.29 (m, 1H, H9). ${ }^{13} \mathrm{C}$ NMR: 41.76, 54.13, 114.45, 118.80, 120.76, 123.35, 126.57, 127.90, 128.65, 129.08, $129.22,134.17,134.41,134.68,142.40,144.31,145.59,152.65$. HRMS (EI) $m / z$ for: $\left[\mathrm{C}_{20} \mathrm{H}_{16} \mathrm{~N}_{6} \mathrm{~S}+\mathrm{H}\right]$ calc. 373.1235 Found 373.1235 .

10-[(1-(4-Fluorobenzyl)-1H-1,2,3-triazol-4-yl)methyl]-1,8-diazaphenothiazine (2c). (142 mg,76\%) yellow oil. ${ }^{1} \mathrm{H} \mathrm{NMR}\left(\mathrm{CDCl}_{3}\right)$ 8:5.32 (s, $\left.2 \mathrm{H}, \mathrm{CH}_{2} \mathrm{~N}_{\mathrm{Th}}\right), 5.46\left(\mathrm{~s}, 2 \mathrm{H}, \mathrm{CH}_{2} \mathrm{~N}_{\mathrm{Tr}}\right), 6.78\left(\mathrm{~m}, 1 \mathrm{H}, \mathrm{H}_{3}\right), 6.79\left(\mathrm{~m}, 1 \mathrm{H}, \mathrm{H}_{6}\right)$, $7.05\left(\mathrm{~m}, 2 \mathrm{H}, 2 \mathrm{H}_{\mathrm{Ph}}\right), 7.19\left(\mathrm{~m}, 1 \mathrm{H}, \mathrm{H}_{4}\right), 7.22\left(\mathrm{~m}, 2 \mathrm{H}, 2 \mathrm{H}_{\mathrm{Ph}}\right), 7.48\left(\mathrm{~s}, 1 \mathrm{H}, \mathrm{H}_{\mathrm{Tr}}\right), 7.96\left(\mathrm{~m}, 1 \mathrm{H}, \mathrm{H}_{2}\right), 8,08(\mathrm{~m}$, $\left.1 \mathrm{H}, \mathrm{H}_{7}\right), 8,28\left(\mathrm{~m}, 1 \mathrm{H}, \mathrm{H}_{9}\right) .{ }^{13} \mathrm{C}$ NMR: 41.83, 53.35, 114.89, 115.73, 115.87, 115.98, 118.59, 123.22, 129.73, $129.78,130.00,130.55,130.57,131.60,134.36,135.79,143.81,144.87,145.39,153.00$. HRMS (EI) $m / z$ for: $\left[\mathrm{C}_{20} \mathrm{H}_{15} \mathrm{FN}_{6} \mathrm{~S}+\mathrm{H}\right]$ calc. 391.1141 Found: 391.1143 .

10-[(1-(4-Chlorobenzyl)-1H-1,2,3-triazol-4-yl)methyl]-1,8-diazaphenothiazine (2d). $140 \mathrm{mg}, 71 \%)$. brown oil. ${ }^{1} \mathrm{H} \mathrm{NMR}\left(\mathrm{CDCl}_{3}\right) \delta: 5.31\left(\mathrm{~s}, 2 \mathrm{H}, \mathrm{CH}_{2} \mathrm{~N}_{\mathrm{Th}}\right), 5.47\left(\mathrm{~s}, 2 \mathrm{H} . \mathrm{CH}_{2} \mathrm{~N}_{\mathrm{Tr}}\right), 6.81\left(\mathrm{~m}, 1 \mathrm{H}, \mathrm{H}_{3}\right)$, $6.93\left(\mathrm{~m}, 1 \mathrm{H}, \mathrm{H}_{6}\right), 7.18\left(\mathrm{~m}, 2 \mathrm{H}, 2 \mathrm{H}_{\mathrm{Ph}}\right), 7.21\left(\mathrm{~m}, 1 \mathrm{H}, \mathrm{H}_{4}\right), 7.34\left(\mathrm{~m}, 2 \mathrm{H}, 2 \mathrm{H}_{\mathrm{Ph}}\right), 7.51\left(\mathrm{~s}, 1 \mathrm{H}, \mathrm{H}_{\mathrm{Tr}}\right), 7.97(\mathrm{~m}, 1 \mathrm{H}$, $\left.\mathrm{H}_{2}\right), 8.04\left(\mathrm{~m}, 1 \mathrm{H}, \mathrm{H}_{7}\right), 8.29\left(\mathrm{~m}, 1 \mathrm{H}, \mathrm{H}_{9}\right) .{ }^{13} \mathrm{C} \mathrm{NMR}: 41.86,53.25,114.49,115.72,115.67,115.98,118.61$, $123.20,129.74,129.76,130.00,130.59,130.47,131.66,134.16,135.69,143.81,144.77,145.49,153.02$. HRMS (EI) $m / z$ for: $\left[\mathrm{C}_{20} \mathrm{H}_{15} \mathrm{ClN}_{6} \mathrm{~S}+\mathrm{H}\right]$ calc. 407.0846 Found: 407.0835 .

10-[(1-(4-Cyanobenzyl)-1H-1,2,3-triazol-4-yl)methyl]-1,8-diazaphenothiazine (2e). (135 mg, 69\%) brown oil. ${ }^{1} \mathrm{H} \mathrm{NMR}\left(\mathrm{CDCl}_{3}\right)$ 8: $5.34\left(\mathrm{~s}, 2 \mathrm{H}, \mathrm{CH}_{2} \mathrm{~N}_{\mathrm{Th}}\right), 5.56\left(\mathrm{~s}, 2 \mathrm{H} . \mathrm{CH}_{2} \mathrm{~N}_{\mathrm{Tr}}\right), 6.80\left(\mathrm{~m}, 1 \mathrm{H}, \mathrm{H}_{3}\right), 6.93\left(\mathrm{~m}, 1 \mathrm{H}, \mathrm{H}_{6}\right)$, $7.21\left(\mathrm{~m}, 1 \mathrm{H}, \mathrm{H}_{4}\right), 7.28\left(\mathrm{~m}, 2 \mathrm{H}, \mathrm{H}_{\mathrm{Ph}}\right), 7.53\left(\mathrm{~s}, 1 \mathrm{H}, \mathrm{H}_{\mathrm{Tr}}\right), 7.65\left(\mathrm{~m}, 2 \mathrm{H}, \mathrm{H}_{\mathrm{Ph}}\right), 7.96\left(\mathrm{~m}, 1 \mathrm{H}, \mathrm{H}_{2}\right), 8.34(\mathrm{~m}, 2 \mathrm{H}$, $\left.\mathrm{H}_{7}+\mathrm{H}_{9}\right) .{ }^{13} \mathrm{C}$ NMR: 41.83, 53.33, 112.64, 114.95, 118.20, 118.68, 123.67, 125.31, 128.24, 129.05, 132.31, $132.86,134.42,135.22,139.91,144.82,145.25,145.41,152.95$. HRMS (EI) $m / z$ for: $\left[\mathrm{C}_{21} \mathrm{H}_{15} \mathrm{~N}_{7} \mathrm{~S}+\mathrm{H}\right]$ calc. 398.1189 Found: 398.1192.

10-[(1-Phenylthiomethyl-1H-1,2,3-triazolo-4-ylo)methyl]-1,8-diazaphenothiazine (2f). (150 mg, 75\%) brown oil. ${ }^{1} \mathrm{H} \mathrm{NMR}\left(\mathrm{CDCl}_{3}\right) \delta: 5.28\left(\mathrm{~s}, 2 \mathrm{H}, \mathrm{CH}_{2} \mathrm{~N}_{\mathrm{Th}}\right), 5.57\left(\mathrm{~s}, 2 \mathrm{H} . \mathrm{CH}_{2} \mathrm{~N}_{\mathrm{Tr}}\right), 6.83\left(\mathrm{~m}, 1 \mathrm{H}, \mathrm{H}_{3}\right), 7.22(\mathrm{~m}$, $\left.1 \mathrm{H}, \mathrm{H}_{6}\right), 7.28\left(\mathrm{~m}, 6 \mathrm{H}, 5 \mathrm{H}_{\mathrm{Ph}}+\mathrm{H}_{4}\right), 7.52\left(\mathrm{~s}, 1 \mathrm{H}, \mathrm{H}_{\mathrm{Tr}}\right), 7.96\left(\mathrm{~m}, 1 \mathrm{H}, \mathrm{H}_{2}\right), 8.07\left(\mathrm{~m}, 1 \mathrm{H}, \mathrm{H}_{7}\right), 8.23(\mathrm{~m}, 1 \mathrm{H}$, $\left.\mathrm{H}_{9}\right) .{ }^{13} \mathrm{C}$ NMR: 41.69, 54.05, 114.39, 118.86, 120.80, 123.02, 128.85, 129.45, 129.53, 129.58, 131.55, 132.54, $132.79,133.15,133.75,133.90,134.45,142.33,144.45,145.65,152.54$. HRMS (EI) $m / z$ for: $\left[\mathrm{C}_{20} \mathrm{H}_{16} \mathrm{~N}_{6} \mathrm{~S}_{2}+\right.$ H] calc. 405.0956 Found: 405.0974 .

10-[(1-Benzyl-1H-1,2,3-triazol-4-yl)-methyl]-2,7-diazaphenothiazine (3b). (142 mg, 79\%) yellow oil.

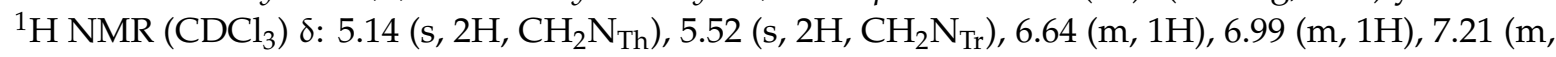
2H), 7,35 (m, 3H), $7.36\left(\mathrm{~s}, 1 \mathrm{H}, \mathrm{H}_{\mathrm{Tr}}\right), 8.01(\mathrm{~m}, 4 \mathrm{H}) .{ }^{13} \mathrm{C}$ NMR: 44.49, 54.40, 109.72, 118.39, 121.95, 126.43, 126.98, 127.58, 128.92, 129.22, 129.33, 133.51, 134.23, 134.41, 141.09, 142.95, 144.83, 146.41. 149.45, 149.87. HRMS (EI) $m / z$ for: $\left[\mathrm{C}_{20} \mathrm{H}_{16} \mathrm{~N}_{6} \mathrm{~S}+\mathrm{H}\right]$ calc. 373.1235 Found373.1241.

10-[(1-(4-Fluorobenzyl)-1H-1,2,3-triazol-4-yl)methyl]-2,7-diazaphenothiazine (3c). (155 mg, 78\%) beige oil. ${ }^{1} \mathrm{H}$ NMR $\left(\mathrm{CDCl}_{3}\right) \delta: 5.13\left(\mathrm{~s}, 2 \mathrm{H}, \mathrm{CH}_{2} \mathrm{~N}_{\mathrm{Th}}\right), 5.47\left(\mathrm{~s}, 2 \mathrm{H}, \mathrm{CH}_{2} \mathrm{~N}_{\mathrm{Tr}}\right), 6.61(\mathrm{~d}, J=5.4 \mathrm{~Hz}, 1 \mathrm{H}), 6.96(\mathrm{~d}$, $J=5.4 \mathrm{~Hz}, 1 \mathrm{H}), 7.02(\mathrm{~m}, 2 \mathrm{H}), 7.19(\mathrm{~m}, 2 \mathrm{H}) ; 7.37\left(\mathrm{~s}, 1 \mathrm{H}, \mathrm{H}_{\mathrm{Tr}}\right), 7.94(\mathrm{~s}, 1 \mathrm{H}), 8.03(\mathrm{~s}, 1 \mathrm{H}), 8.07(\mathrm{~d}, J=5.4 \mathrm{~Hz}$, 1H), 8.12 (d, $J=5.4 \mathrm{~Hz}, 1 \mathrm{H}) .{ }^{13} \mathrm{C}$ NMR: 44.38, 53.63, 109.61, 116.16, 116.30, 121.57, 121.95, 121.86, 129.77, 
129.83, 130.08, 130.10, 133.55, 135.40, 138.02, 138.09, 143.09, 144.83, 146.56. 149.57, 149.79. HRMS (EI) $m / z$ for: $\left[\mathrm{C}_{20} \mathrm{H}_{15} \mathrm{FN}_{6} \mathrm{~S}+\mathrm{H}\right]$ calc. 391.1141 Found: 391.1140 .

10-[(1-(4-Chlorobenzyl)-1H-1,2,3-triazol-4-yl)methyl]-2,7-diazaphenothiazine (3d). (154 mg, 82\%) beige oil. ${ }^{1} \mathrm{H}$ NMR $\left(\mathrm{CDCl}_{3}\right) \delta: 5.13\left(\mathrm{~s}, 2 \mathrm{H}, \mathrm{CH}_{2} \mathrm{~N}_{\mathrm{Th}}\right) ; 5.49\left(\mathrm{~s}, 2 \mathrm{H}, \mathrm{CH}_{2} \mathrm{~N}_{\mathrm{Tr}}\right), 6.62(\mathrm{~m}, 1 \mathrm{H}), 6.97(\mathrm{~m}, 1 \mathrm{H}), 7.17(\mathrm{~m}$, 2H), $7.35(\mathrm{~m}, 2 \mathrm{H}), 7,39\left(\mathrm{~s}, 1 \mathrm{H}, \mathrm{H}_{\mathrm{Tr}}\right), 8,14(\mathrm{~m}, 4 \mathrm{H}) .{ }^{13} \mathrm{C}$ NMR: 44.49, 53.69, 109.73, 117.39, 121.57, 121.91, 129.26, 129.46, 132.64, 133.60, 135.05, 135.32, 138.09, 142.98, 144.90, 145.88, 149.01, 150.19. HRMS (EI) $m / z$ for: $\left[\mathrm{C}_{20} \mathrm{H}_{15} \mathrm{ClN}_{6} \mathrm{~S}+\mathrm{H}\right]$ calc. 407.0846 Found: 407.0855 .

10-[(1-(4-Cyanobenzyl)-1H-1,2,3-triazol-4-yl)methyl]-2,7-diazaphenothiazine (3e). (138 mg, 71\%) brown oil. ${ }^{1} \mathrm{H}$ NMR $\left(\mathrm{CDCl}_{3}\right)$ 8: $5.11\left(\mathrm{~s}, 2 \mathrm{H}, \mathrm{CH}_{2} \mathrm{~N}_{\mathrm{Th}}\right), 5.59\left(\mathrm{~s}, 2 \mathrm{H}, \mathrm{CH}_{2} \mathrm{~N}_{\mathrm{Tr}}\right) ; 6.66(\mathrm{~m}, 1 \mathrm{H}), 6.97(\mathrm{~m}, 1 \mathrm{H}), 7.31(\mathrm{~m}$, 2H), $7.46\left(\mathrm{~s}, 1 \mathrm{H}, \mathrm{H}_{\mathrm{Tr}}\right), 8.04(\mathrm{~m}, 1 \mathrm{H}), 8.17(\mathrm{~m}, 3 \mathrm{H}) .{ }^{13} \mathrm{C}$ NMR: 44.27, 53.64, 113.03, 118.39, 120.57, 121.37, 122.11, 122.30, 122.41, 132.87, 133.00, 137.60, 139.32, 143.33, 143.46, 144.12, 145.75, 146.20, 148.55, 149.19, 152.12. HRMS (EI) $m / z$ for: $\left[\mathrm{C}_{21} \mathrm{H}_{15} \mathrm{~N}_{7} \mathrm{~S}+\mathrm{H}\right]$ calc. 398.1189 Found: 398.1187.

10-[(1-Phenylthiomethyl-1H-1,2,3-triazolo-4-ylo)methyl]-2,7-diazaphenothiazine (3f). (155 mg, 79\%) brown oil. ${ }^{1} \mathrm{H}$ NMR $\left(\mathrm{CDCl}_{3}\right) \delta: 5.12\left(\mathrm{~s}, 2 \mathrm{H}, \mathrm{CH}_{2} \mathrm{~N}_{\mathrm{Th}}\right), 5.59\left(\mathrm{~s}, 2 \mathrm{H}, \mathrm{CH}_{2} \mathrm{~N}_{\mathrm{Tr}}\right), 6.59(\mathrm{~m}, 1 \mathrm{H}), 7.04(\mathrm{~m}, 1 \mathrm{H})$, $7.24(\mathrm{~m}, 2 \mathrm{H}), 7.29$ (m. 3H), 7.35 (s, $\left.1 \mathrm{H}, \mathrm{H}_{\mathrm{Tr}}\right), 8.18(\mathrm{~m}, 4 \mathrm{H}) .{ }^{13} \mathrm{C}$ NMR: 44.36, 54.34, 109.81, 118.37, 121.63, $126.43,126.98,127.58,128.54,129.22,129.33,133.51,134.23,134.41,135.31,142.85,144.90,146.13 .149 .13$, 149.89. HRMS (EI) $m / z$ for: $\left[\mathrm{C}_{20} \mathrm{H}_{16} \mathrm{~N}_{6} \mathrm{~S}_{2}+\mathrm{H}\right]$ calc. 405.0956 Found: 405.0963.

10-[(1-Benzyl-1H-1,2,3-triazol-4-yl)-methyl]-3,6-diazaphenothiazine $(4 \mathbf{b}) .(144 \mathrm{mg}, 80 \%)$ yellow oil. ${ }^{1} \mathrm{H}$ $\operatorname{NMR}\left(\mathrm{CDCl}_{3}\right)$ 8: $5.07\left(\mathrm{~s}, 2 \mathrm{H}, \mathrm{CH}_{2} \mathrm{~N}_{\mathrm{Th}}\right), 5.27\left(\mathrm{~s}, 2 \mathrm{H}, \mathrm{CH}_{2} \mathrm{~N}_{\mathrm{Tr}}\right), 6.62(\mathrm{~m}, 1 \mathrm{H}), 6.94(\mathrm{~m}, 1 \mathrm{H}), 6.98(\mathrm{~m}, 1 \mathrm{H})$, $7.21\left(\mathrm{~m}, 2 \mathrm{H}, \mathrm{H}_{\mathrm{Ph}}\right), 7.36\left(\mathrm{~m}, 3 \mathrm{H}, \mathrm{H}_{\mathrm{Ph}}\right), 7.37\left(\mathrm{~s}, 1 \mathrm{H}, \mathrm{H}_{\mathrm{Tr}}\right), 8.03(\mathrm{~m}, 1 \mathrm{H}), 8.19(\mathrm{~m}, 2 \mathrm{H}) .{ }^{13} \mathrm{C}$ NMR: 44.52, 54.45, 109.31, 121.40, 122.04, 122.12, 124.97, 126.39, 128.01, 128.87, 129.34, 130.44, 134.21, 137.68, 142.95, 144.03, 145.63, 145.96, 148.34, 149.25. HRMS (EI) $m / z$ for: $\left[\mathrm{C}_{20} \mathrm{H}_{16} \mathrm{~N}_{6} \mathrm{~S}+\mathrm{H}\right]$ calc. 373.1235 Found 373.1229.

10-[(1-(4-Fluorobenzyl)-1H-1,2,3-triazol-4-yl)methyl]-3,6-diazaphenothiazine (4c). (154 mg, 82\%) beige oil. ${ }^{1} \mathrm{H}$ NMR $\left(\mathrm{CDCl}_{3}\right) \delta: 5.03\left(\mathrm{~s}, 2 \mathrm{H}, \mathrm{CH}_{2} \mathrm{~N}_{\mathrm{Th}}\right), 5.47\left(\mathrm{~s}, 2 \mathrm{H}, \mathrm{CH}_{2} \mathrm{~N}_{\mathrm{Tr}}\right), 6.53(\mathrm{~m}, 1 \mathrm{H}), 6.89(\mathrm{~m}, 2 \mathrm{H}), 7.05(\mathrm{~m}$, $\left.2 \mathrm{H}, \mathrm{H}_{\mathrm{Ph}}\right), 7.19\left(\mathrm{~m}, 2 \mathrm{H}, \mathrm{H}_{\mathrm{Ph}}\right), 7.39\left(\mathrm{~s}, 1 \mathrm{H}, \mathrm{H}_{\mathrm{Tr}}\right), 7.95(\mathrm{~m}, 1 \mathrm{H}), 8.05(\mathrm{~m}, 1 \mathrm{H}), 8.10(\mathrm{~m}, 1 \mathrm{H}) .{ }^{13} \mathrm{C} \mathrm{NMR}: 44.33$, 53.63, 109.09, 115.84, 116.32, 118.68, 121.66, 122.04, 129.84, 130.14, 137.79, 143.19, 143.76, 145.70, 146.81, 148.76, 148.87, 149.09. HRMS (EI) $\mathrm{m} / z$ for: $\left[\mathrm{C}_{20} \mathrm{H}_{15} \mathrm{FN}_{6} \mathrm{~S}+\mathrm{H}\right]$ calc. 391.1141 Found: 391.1140.

10-[(1-(4-Chlorobenzyl)-1H-1,2,3-triazol-4-yl)methyl]-3,6-diazaphenothiazine (4d). (150 mg, 76\%) beige oil. ${ }^{1} \mathrm{H}$ NMR $\left(\mathrm{CDCl}_{3}\right) \delta: 5.07\left(\mathrm{~s}, 2 \mathrm{H}, \mathrm{CH}_{2} \mathrm{~N}_{\mathrm{Th}}\right), 5.48\left(\mathrm{~s}, 2 \mathrm{H}, \mathrm{CH}_{2} \mathrm{~N}_{\mathrm{Tr}}\right), 6.59(\mathrm{~m}, 1 \mathrm{H}), 6.93\left(\mathrm{~m}, 2 \mathrm{H}, \mathrm{H}_{\mathrm{Ph}}\right)$, $7.16\left(\mathrm{~m}, 2 \mathrm{H}, \mathrm{H}_{\mathrm{Ph}}\right), 7.39\left(\mathrm{~s}, 1 \mathrm{H}, \mathrm{H}_{\mathrm{Tr}}\right), 7.95(\mathrm{~m}, 1 \mathrm{H}), 8.02(\mathrm{~m}, 1 \mathrm{H}), 8.10(\mathrm{~m}, 1 \mathrm{H}) .{ }^{13} \mathrm{C}$ NMR: 44.21, 53.64, 109.19, 115.64, 116.22, 118.68, 121.86, 122.14, 129.94, 130.24, 137.89, 143.39, 143.96, 145.80, 146.85, 148.66, 148.97, 149.19. HRMS (EI) $\mathrm{m} / z$ for: $\left[\mathrm{C}_{20} \mathrm{H}_{15} \mathrm{ClN}_{6} \mathrm{~S}+\mathrm{H}\right]$ calc. 407.0846 Found: 407.0837.

10-[(1-(4-Cyanobenzyl)-1H-1,2,3-triazol-4-yl)methyl]-3,6-diazaphenothiazine (4e). (139 $\mathrm{mg}, 72 \%)$ brown oil. ${ }^{1} \mathrm{H}$ NMR $\left(\mathrm{CDCl}_{3}\right) \delta: 5.11\left(\mathrm{~s}, 2 \mathrm{H}, \mathrm{CH}_{2} \mathrm{~N}_{\mathrm{Th}}\right), 5.59\left(\mathrm{~s}, 2 \mathrm{H}, \mathrm{CH}_{2} \mathrm{~N}_{\mathrm{Tr}}\right), 6.63(\mathrm{~m}, 1 \mathrm{H}), 6.96(\mathrm{~m}, 2 \mathrm{H}), 7.32(\mathrm{~m}$, $\left.2 \mathrm{H}, \mathrm{H}_{\mathrm{Ph}}\right), 7.48\left(\mathrm{~s}, 1 \mathrm{H}, \mathrm{H}_{\mathrm{Tr}}\right), 7.67\left(\mathrm{~m}, 2 \mathrm{H}, \mathrm{H}_{\mathrm{Ph}}\right), 8.05(\mathrm{~m}, 1 \mathrm{H}), 8.14(\mathrm{~m}, 2 \mathrm{H}) .{ }^{13} \mathrm{C}$ NMR: 44.27, 53.65, 109.21, 113.05, 117.99, 121.36, 122.07, 122.11, 127.88, 128.01, 128.52, 132.83, 132.87, 133.02, 135.58, 137.60, 139.30, 143.47, 144.12, 148.34, 149.25. HRMS (EI) $m / z$ for: $\left[\mathrm{C}_{21} \mathrm{H}_{15} \mathrm{~N}_{7} \mathrm{~S}+\mathrm{H}\right]$ calc. 398.1189 Found: 398.1184 .

10-[(1-Phenylthiomethyl-1H-1,2,3-triazolo-4-ylo)methyl]-3,6-diazaphenothiazine (4f). (153 mg, 78\%) brown oil. ${ }^{1} \mathrm{H}$ NMR $\left(\mathrm{CDCl}_{3}\right) \delta: 5.05\left(\mathrm{~s}, 2 \mathrm{H}, \mathrm{CH}_{2} \mathrm{~N}_{\mathrm{Th}}\right), 5.62\left(\mathrm{~s}, 2 \mathrm{H}, \mathrm{CH}_{2} \mathrm{~N}_{\mathrm{Tr}}\right), 6.55(\mathrm{~m}, 1 \mathrm{H}), 6.93(\mathrm{~m}, 1 \mathrm{H})$, $6.97(\mathrm{~m}, 1 \mathrm{H}), 7.24(\mathrm{~m}, 5 \mathrm{H}), 7.33\left(\mathrm{~s}, 1 \mathrm{H}, \mathrm{H}_{\mathrm{Tr}}\right), 8.07(\mathrm{~m}, 1 \mathrm{H}), 8.15(\mathrm{~m}, 2 \mathrm{H}) .{ }^{13} \mathrm{C}$ NMR: 44.45, 54.26, 109.01, $121.43,121.72,122.21,129.08,129.58,131.21,132.52,137.37,142.66,144.33,145.01,145.38,147.62,149.76$. HRMS (EI) $m / z$ for: $\left[\mathrm{C}_{20} \mathrm{H}_{16} \mathrm{~N}_{6} \mathrm{~S}_{2}+\mathrm{H}\right]$ calc. 405.0956 Found: 405.0955 .

\subsection{Anticancer Effects In Vitro}

\subsubsection{Cell Culture}

All dipyridothiazines with 1,2,3-triazole substituents (1-4)b-f were evaluated for their anticancer activity using four cultured cell lines: SNB-19 (human glioblastoma, DSMZ-German Collection of Microorganisms and Cell Cultures, Braunschweig, Germany), Caco-2 (human colon adenocarcinoma, DSMZ-German Collection of Microorganisms and Cell Cultures, Braunschweig, Germany), 
A549 (human lung carcinoma, ATCC, Manassas, VA, USA) and MDA-MB231 (human breast adenocarcinoma, ATCC, Manassas, VA, USA), and NHDF (human dermal fibroblast cell line, ATCC, Manassas, VA, USA. The cultured cells were kept at $37^{\circ} \mathrm{C}$ and $5 \% \mathrm{CO}_{2}$. The cells were seeded $(1 \times$ $10^{4}$ cells/well/100 $\mu \mathrm{L}$ DMEM supplemented with 10\% FCS and streptomycin and penicillin) using 96-well plates (Corning). The cells were counted in a hemocytometer (Burker's chamber) using a phase contrast Olympus IX50 microscope equipped with Sony SSC-DC58 AP camera and Olympus DP10 digital camera.

\subsubsection{Cell Proliferation and Viability}

Antiproliferative effect of the obtained compounds on the cancer cells was determined using the cell proliferation reagent WST-1 assay (Roche Diagnostics, Mannheim, Germany). This assay is based on the viable cell's ability to cleave the bright red-colored tetrazolium salt (2-(4-iodophenyl)-3-(4-nitrophenyl)-5-(2,4-disulfophenyl)-2H-tetrazolium, monosodium salt) to dark red soluble formazan by cellular enzymes. An increase in the amount of formazan dye formed correlates to the number of metabolically active cells in the culture. The formazan dye produced by metabolically active cells is quantified by a scanning ELISA reader by measuring the absorbance of the dye solution at appropriate wavelengths. The examined cells were exposed to the tested compounds for $72 \mathrm{~h}$ at various concentrations between $0.1 \mu \mathrm{g} / \mathrm{mL}$ and $100 \mu \mathrm{g} / \mathrm{mL}$ (prepared initially at concentration of $1 \mathrm{mg} / \mathrm{mL} \mathrm{in}$ DMSO). The cells were incubated with WST-1 $(10 \mu \mathrm{L})$ for $1 \mathrm{~h}$ and the absorbance of the samples against a background control was measured at $450 \mathrm{~nm}$ using a microplate reader with a reference wavelength at $600 \mathrm{~nm}$. The results are expressed as means of at least two independent experiments performed in triplicate. The antiproliferative activity of the tested compound was compared to cisplatin. The IC 50 values (a concentration of a compound that is required for $50 \%$ inhibition) were calculated from the dose-response relationship with respect to control.

\subsubsection{The RT-qPCR Method}

Genes trancriptional activity (H3, TP53, CDKN1A, BCL-2, and BAX) was evaluated by real time RT-qPCR with OPTICON TM DNA Engine (MJ Research, Watertown, MA, USA) and QuantTect ${ }^{\circledR}$ SYBR $^{\circledR}$ Green RT-PCR Kit (Quiagen, Valencia, CA). Cells were exposed to compound 4 at a $0.5 \mu \mathrm{g} / \mathrm{mL}$ concentration for $24 \mathrm{~h}$. The RNA extraction was made by using Quick-RNA ${ }^{\mathrm{TM}}$ Kit MiniPrep (ZYMO RESEARCH). Total RNA integrity was analysed in $1.2 \%$ agarose electrophoresis with added ethidium bromide. The quantity and purity of extracted total RNA were determined by using spectrophotometric analysis with HP845 (Hewlett Packard, Waldbronn, Germany) spectrophotometer. The statistical analysis was performed using the Statistica 8.0 software. All values were expressed as means \pm SE.

\section{Conclusions}

We report here efficient synthesis of a new isomeric series of novel 1,2,3-triazole-dipyridothiazine hybrids in the 1,3-dipolar cycloadditon reactions. The structure of these new compounds was identified using advanced two-dimensional ${ }^{1} \mathrm{H}$ and ${ }^{13} \mathrm{C}$ NMR (COSY, ROESY) spectra. Most of the obtained derivatives of diazaphenothiazines exhibited significant anti-proliferative activities against the human glioblastoma SNB-19, colorectal carcinoma Caco-2, breast cancer MDA-MB-231 and lung cancer A549 cell lines with the $\mathrm{IC}_{50}$ values $<1 \mu \mathrm{M}$ and were more potent than cisplatin. The most promising compound 1d was used for gene expression analysis by the RT-qPCR method. The expression of $H 3$, TP53, CDKN1A, BCL-2, and BAX genes revealed that this compound inhibited the proliferation in all cells (H3) and activated mitochondrial events of apoptosis (BCL-2/BAX) in MDA-MB231 cancer cell line. Further in vitro and in vivo studies are required to determine the potential pharmacological use of these dipyrido-1,2,3-triazole hybrids in anticancer therapy. 
Supplementary Materials: The supplementary materials are available online.

Author Contributions: B.M.-M. and K.P. developed the concept of the work. B.M.-M. carried out the synthetic work and interpreted the results. M.J. contributed to the synthesis and purification selected compounds. M.L. and D.K. carried out the biological experiments and interpreted the results. All authors have given approval to the final version of the manuscript.

Funding: This research was funded by the Medical University of Silesia in Katowice, grant KNW-1-055/K/9/O.

Acknowledgments: The work was supported by the Medical University of Silesia in Katowice (Grant KNW-1-055/K/9/O.).

Conflicts of Interest: The authors declare no conflict of interest.

\section{References}

1. Mitchell, S.C. Phenothiazine: The Parent molecule. Curr. Drug Targ. 2006, 7, 1181-1189. [CrossRef] [PubMed]

2. Amaral, L.; Viveiros, M. Thioridazine: A Non-Antibiotic Drug Highly Effective, in Combination with First Line Anti-Tuberculosis Drugs, against Any Form of Antibiotic Resistance of Mycobacterium tuberculosis Due to Its Multi-Mechanisms of Action. Antibiotics 2017, 6, 3. [CrossRef] [PubMed]

3. Yue, H.; Huang, D.; Qin, L.; Zheng, Z.; Hua, L.; Wang, G.; Huang, J.; Huang, H. Targeting Lung Cancer Stem Cells with Antipsychological Drug Thioridazine. BioMed Res. Int. 2016, 2016, 6709828. [CrossRef] [PubMed]

4. Mosnaim, A.D.; Ranade, V.V.; Wolf, M.E. Phenothiazine molecule provides the basic chemical structure for various classes of pharmacotherapeutic agents. Am. J. Ther. 2006, 13, 261-273. Available online: https://journals.lww.com/americantherapeutics/toc/2006/05000 (accessed on 12 June 2019). [CrossRef]

5. Dasgupta, A.; Dastridara, S.G.; Shirataki, Y.; Motohashi, N. Antibacterial activity of artificial phenothiazines and isoflavones from plants. Top Heterocycl. Chem. 2008, 15, 67-132.

6. Aaron, J.J.; Gaye Seye, M.D.; Trajkovska, S.; Motohashi, N. Bioactive Phenothiazines and Benzo[a]phenothiazines: Spectroscopic studies and biological and biomedical properties and applications. Top Heterocycl. Chem. 2009, 16, 153-231.

7. Pluta, K.; Morak-Młodawska, B.; Jeleń, M. Synthesis and properties of diaza-, triaza- and tetraazaphenothiazines. J. Heterocycl. Chem. 2009, 46, 355-391. [CrossRef]

8. Pluta, K.; Jeleń, M.; Morak-Młodawska, B.; Zimecki, M.; Artym, J.; Kocięba, M.; Zaczyńska, E. Azaphenothiazines a promising phenothiazine derivatives. An insight into nomenclature, synthesis, structure elucidation and biological properties. Eur. J. Med. Chem. 2017, 138, 774-806. [CrossRef]

9. Pluta, K.; Morak-Młodawska, B.; Jeleń, M. Recent progress in biological activities of synthesized phenothiazines. Eur. J. Med. Chem. 2011, 46, 3179-3189. [CrossRef]

10. Jaszczyszyn, A.; Gąsiorowski, K.; Świątek, P.; Malinka, W.; Cieślik-Boczula, K.; Petrus, J.; Czarnik-Matusewicz, B. Chemical structure of phenothiazines and their biological activity. Pharmacol. Rep. 2012, 64, 16-23. Available online: http://www.if-pan.krakow.pl/pjp/pdf/2012/1_16.pdf (accessed on 12 June 2019). [CrossRef]

11. Jeleń, M.; Pluta, K.; Latocha, M.; Morak-Młodawska, B.; Suwińska, K.; Kusmierz, D. Evaluation of angularly condensed diquinothiazines as potential anticancer agents. Bioorg. Chem. 2019, 87, 810-820. [CrossRef]

12. González-Muñoz, G.C.; Arce, M.P.; López, B.; Pérez, C.; Villarroya, M.; López, M.G.; García, A.G.; Conde, S.; Rodríguez-Franco, M.I. Old phenothiazine and dibenzothiadiazepine derivatives for tomorrow's neuroprotective therapies against neurodegenerative diseases. Eur. J. Med. Chem. 2010, 45, 6152-6158. [CrossRef] [PubMed]

13. Strządala, L.; Fiedorowicz, A.; Wysokińska, E.; Zioło, E.; Grudzień, M.; Jeleń, M.; Pluta, K.; Morak-Młodawska, B.; Zimecki, M.; Kałas, W. An Anti-Inflammatory Azaphenothiazine Inhibits Interferon $\beta$ Expression and CXCL10 Production in KERTr Cells. Molecules 2018, 23, 2433. [CrossRef] [PubMed]

14. Presti, M.; Bazán, P.; Strauss, M.; Báez, A.; Rivarola, H.; Paglini-Oliva, P. Trypanothione reductase inhibitors: Overview of the action of thioridazine in different stages of Chagas disease. Acta Trop. 2015, 145, 79-87. [CrossRef]

15. Spengler, G.; Csonka, A.; Molnar, J.; Amaral, L. The anticancer activity of the old neuroleptic phenothiazine-type drug thioridazine. Anticancer Res. 2016, 36, 5701-5706. [CrossRef] 
16. Liu, N.; Jin, Z.; Zhang, J.; Jin, J. Antitumor evaluation of novel phenothiazine derivatives that inhibit migration and tubulin polymerization against gastric cancer MGC-803 cells. Investig. New Drugs 2018, 37, 188-198. [CrossRef]

17. Pluta, K.; Jeleń, M.; Morak-Młodawska, B.; Zimecki, M.; Artym, J.; Kocięba, M. Anticancer activity of newly synthesized azaphenothiazines in NCI's anticancer screening. Pharmacol. Rep. 2010, 62, 319-332. Available online: http://www.if-pan.krakow.pl/pjp/pdf/2010/2_319.pdf (accessed on 12 June 2019). [CrossRef]

18. Morak-Młodawska, B.; Pluta, K.; Matralis, A.N.; Kourounakis, A.P. Antioxidant Activity of Newly Synthesized 2, 7-Diazaphenothiazines. Arch. Pharm. Int. J. Pharm. Med. Chem. 2010, 343, 268-273. [CrossRef]

19. Zimecki, M.; Artym, J.; Kocięba, M.; Pluta, K.; Morak-Młodawska, B.; Jeleń, M. Immunosupressive activities of newly synthesized azaphenothiazines in human and mouse models. Cell. Mol. Biol. Lett. 2009, 14, 622-635. [CrossRef]

20. Morak-Młodawska, B.; Pluta, K.; Zimecki, M.; Jeleń, M.; Artym, J.; Kocięba, M. Synthesis and selected immunological properties of 10-substituted 1, 8-diazaphenothiazines. Med. Chem. Res. 2015, 24, 1408-1418. [CrossRef]

21. Morak-Młodawska, B.; Pluta, K.; Latocha, M.; Jeleń, M.; Kuśmierz, D. Synthesis and anticancer and lipophilic properties of 10-dialkylaminobutynyl derivatives of 1, 8- and 2, 7-diazaphenothiazines. J. Enzyme Inhib. Med. Chem. 2016, 31, 1132-1138. [CrossRef] [PubMed]

22. Morak-Młodawska, B.; Pluta, K.; Latocha, M.; Jeleń, M. Synthesis, spectroscopic characterization, and anticancer activity of new 10-substituted 1, 6-diazaphenothiazines. Med. Chem. Res. 2016, 25, 2425-2433. [CrossRef] [PubMed]

23. Morak-Młodawska, B.; Pluta, K.; Latocha, M.; Suwińska, K.; Jeleń, M.; Kuśmierz, D. 3, 6-Diazaphenothiazines as potential lead molecules-synthesis, characterization and anticancer activity. J. Enzyme Inhib. Med. Chem. 2016, 31, 1512-1519. [CrossRef] [PubMed]

24. Zhang, J.; Chen, M.; Wenzhi, Z.; Okechukwu, P.N.; Morak-Młodawska, B.; Pluta, K.; Jeleń, M.; Md Akim, A.; Ang, K.-P.; Ooi, K.K. 10H-3, 6-Diazaphenothiazines Induce G2/M Phase Cell Cycle Arrest, Caspase-dependent Apoptosis and Inhibits Cell Invasion of A2780 Ovarian Carcinoma Cells through Regulation on NF- $\mathrm{B}$ and [BIRC6-XIAP] Complexes. Drug Des. Dev. Ther. 2017, 11, 3045-3063. [CrossRef] [PubMed]

25. Morak-Młodawska, B.; Pluta, K.; Latocha, M.; Jeleń, M.; Kuśmierz, D. Synthesis, anticancer activity and apoptosis induction of novel 3, 6-diazaphenothiazines. Molecules 2019, 24, 267. [CrossRef]

26. Dheer, D.; Singh, V.; Shankar, R. Medicinal attributes of 1, 2, 3-triazoles: Current developments. Bioorg. Chem. 2017, 71, 30-54. [CrossRef]

27. Duan, Y.C.; Ma, Y.C.; Zhang, E.; Shi, X.J.; Wang, M.M.; Ye, X.W.; Liu, H.M. Design and synthesis of novel 1, 2, 3-triazole-dithiocarbamate hybrids as potential anticancer agents. Eur. J. Med. Chem. 2013, 62, 11-19. [CrossRef]

28. Totobennazara, J.; Burke, J.A. New click-chemistry methods for 1, 2, 3-triazoles synthesis: Recent advances and applications. Tetrahedron Lett. 2015, 56, 2853-2859. [CrossRef]

29. Sharma, P.; Kumar, A.; Upadhyay, S.; Singh, J.; Sahu, V. A novel approach to the synthesis of 1, 2, 3-triazoles and their SAR studies. Med. Chem. Res. 2010, 19, 589-602. [CrossRef]

30. Vatmurge, N.S.; Hazra, B.G.; Pore, V.S.; Shirazi, F.; Chavan, P.S.; Deshpande, M.V. Synthesis and antimicrobial activity of lactam-bile acid conjugates linked via triazole. Bioorg. Med. Chem. Lett. 2008, 18, 2043-2047. [CrossRef]

31. Thirumurugan, P.; Matosiuk, D.; Jóźwiak, K. Click Chemistry for Drug Development and Diverse Chemical-Biology Application. Chem. Rev. 2013, 113, 4905-49792. [CrossRef] [PubMed]

32. Sharma, J.; Ahmad, S.; Alam, M.S. Bioactive triazoles: A potential review. J. Chem. Pharm. Res. 2012, 4, 5157-5164.

33. Keri, R.S.; Patil, S.A.; Budagumpi, S.; Nagaraja, B.M. Triazole: Apromising antitubercular agent. Chem. Biol. Drug Des. 2015, 86, 410-423. [CrossRef] [PubMed]

34. McNulty, J.; Keskar, K. A Robust, Well-Defined Homogeneous Silver(I) Catalyst for MildIntramolecular Hydroamination of 2-Ethynylanilines Leading to Indoles. Eur. J. Org. Chem. 2014, 2014, 1622-1629. [CrossRef]

35. Allen, M.A.; Andrysik, Z.; Dengler, V.L.; Mellert, H.S.; Guarnieri, A.; Freeman, J.A.; Sullivan, K.D.; Galbraith, M.D.; Luo, X.; Dowell, R.D.; et al. Global analysis of p53-regulated transcription identifies its direct targets and unexpected regulatory mechanisms. Elife 2014, 3, 1-29. [CrossRef] 
36. Vander Heiden, M.G.; Cantley, L.C.; Thompson, C.B. Understanding the Warburg Effect: The Metabolic Requirements of Cell Proliferation. Science 2009, 324, 1029-1033. [CrossRef]

37. Beberok, A.; Wrześniok, D.; Rok, J.; Rzepka, Z.; Respondek, M.; Buszman, E. Ciprofloxacin triggers the apoptosis of human triple-negative breast cancer MDA-MB-231 cells via the p53/Bax/Bcl-2 signaling pathway. Int. J. Oncol. 2018, 52, 1727-1737. [CrossRef]

38. Beberok, A.; Rzepka, Z.; Respondek, M.; Rok, J.; Sierotowicz, D.; Wrześniok, D. GSH depletion, mitochondrial membrane breakdown, caspase-3/7 activation and DNA fragmentation in U87MG glioblastoma cells: New insight into the mechanism of cytotoxicity induced by fluoroquinolones. Eur. J. Pharmacol. 2018, 835, 94-107. [CrossRef]

39. Dutto, I.; Tillhon, M.; Cazzalini, O.; Stivala, L.A.; Prosperi, E. Biology of the cell cycle inhibitor p21 CDKN1A: Molecular, mechanisms and relevance in chemical toxicology. Arch. Toxicol. 2015, 89, 155-178. [CrossRef]

40. Elmore, S. Apoptosis: A review of programmed cell death. Toxicol. Pathol. 2007, 35, 495-516. [CrossRef]

41. Hemann, M.T.; Lowe, S.W. The p53-Bcl-2 connection. Cell Death Differ. 2006, 13, 1256-1259. [CrossRef] [PubMed]

Sample Availability: Samples of the compounds. $\mathbf{1 b}-\mathbf{4 f}$. are available from the authors. 